\title{
Relato de experiência sobre a elaboração e construção de vídeo-aula para o ensino do exame físico respiratório
}

\author{
Experience report on the elaboration and construction of a video lesson for the teaching of \\ the respiratory physical exam
}
Informe de experiencia sobre la elaboración y construcción de una video lección para la enseñanza del examen físico respiratorio

Carolina de Freitas Cortes $^{1 *}$, Nathalia Amado da Silva Medeiros ${ }^{1}$, Patrícia Costa dos Santos da Silva1, Suely Amorim de Araújo1, Lívia Ferreira Oliveira¹, Elias Rodrigues de Almeida Júnior ${ }^{1}$, Kleber Gontijo de Deus², Dênis Willian de Oliveira Dias ${ }^{1}$.

\section{RESUMO}

Objetivo: Descrever a elaboração e a construção de um vídeo de simulação para o ensino do exame físico respiratório. Relato de experiência: estudo descritivo, metodológico sobre a elaboração e construção de um vídeo de simulação para o ensino do exame físico. Foi realizada uma busca de literatura para selecionar o referencial teórico para construção dos passos necessários para o exame físico respiratório. 0 vídeo foi elaboração para ser utilizado no debrifieng durante as aulas de sistematização da assistência de enfermagem, como material de reflexão. Como potencialidades destacam-se: as imagens visuais permitem assinalar com mais clareza os conteúdos simulados em cenário realístico, proporcionam condições para o diálogo e reflexões realizadas pelo grupo. Considerações finais: O processo que foi percorrido possibilitou o estímulo do raciocínio clínico, além de permitir que os estudantes e os profissionais executem o exame físico e o processo de enfermagem em diferentes cenários e em espaços protegidas.

Palavras-chave: Enfermagem, Simulação, Educação em Enfermagem.

\begin{abstract}
Objective: To describe the elaboration and construction of a simulation video for respiratory physical examination teaching. Experience report: descriptive and methodological study about the elaboration and construction of a simulation video for physical examination teaching. A literature search was carried out to select the theoretical reference for the construction of the necessary steps for respiratory physical examination. The video was designed to be used during debriefing for nursing care systematization classes as a reflection material. As highlighted potentialities we have:the visual images allow to indicate more clearly the simulated contents in a realistic scenario, besides providing conditions for the dialogue and reflections carried out by the group. Final considerations: The process allowed clinical reasoning stimulation, besides allowing students and professionals to perform the physical examination and the nursing process in different scenarios and protected spaces.
\end{abstract}

Keywords: Nursing, Simulation, Education in Nursing.

\section{RESUMEN}

Objetivo: Describir la elaboración y construcción de un video de simulación para la enseñanza del examen físico respiratorio. Informe de experincia: Enseñanza descriptiva, metodológica sobre la elaboración y construcción de un video de simulación para la enseñanza del examen físico. Se realizó una búsqueda bibliográfica para seleccionar la referencia teórica para la construcción de los pasos necesarios para el ejercicio físico respiratorio. El video fue diseñado para ser utilizado durante las clases del sistema de atención de enfermería, como material de reflexión. A medida que se destacan las potencialidades: a

\footnotetext{
1Universidade Federal de Uberlândia (UFU), Uberlândia- Minas Gerais. *E-mail: caarolfcortes@gmail.com

${ }^{2}$ Hospital de Clínicas de Uberlândia (HCU -UFU), Uberlândia- Minas Gerais.
} 
medida que las imágenes visuales apuntaban con mayor claridad a los índices simulados en un escenario realista, se proporcionan las condiciones para el diálogo y las reflexiones realizadas por el grupo. Consideraciones finales: El proceso que se llevó a cabo permitió al Estímulo realizar un razonamiento clínico, además de permitir que los Estudiantes y Profesionales realicen el Examen Físico y el Proceso de Enfermería en Diferentes Escenarios en y en Espacios Protegidos.

Palabras clave: Enfermería, Simulación, Educación en Enfermería.

\section{INTRODUÇÃO}

A difusão da tecnologia e da informática estimulou a utilização de estratégias de ensino mais arrojadas e que permitam o desenvolvimento da autonomia individual, proporcionando a interdependência e a transdisciplinaridade (DE CARVALHO SANINO GE, 2012; OLIVEIRA SND, et al., 2014; ALMEIDA RGDS, et al., 2015). A apropriação destas estratégias virtuais no ensino da enfermagem se justifica pela informação complexa e em grande quantidade que precisa ser utilizada nos processos de tomada de decisão (OLIVEIRA SND, et al., 2014).

Atualmente, o grande avanço tecnológico e científico tem repercutido na área da saúde, especialmente na enfermagem, por meio de informações que são processadas em uma velocidade extraordinária, o que aponta para a necessidade de atualização e apropriação de ferramentas metodológicas de ensino que possam acompanhar tal evolução do mundo contemporâneo. Nesse sentido, as metodologias ativas de ensino e aprendizagem propõem a formação de um aluno crítico, reflexo e conectado com as inovações científicas (OLIVEIRA SND, et al., 2014).

$O$ uso da simulação no ensino da enfermagem tem se mostrado uma estratégia que estimula a criatividade e o raciocínio clínico dos alunos (JOHNSEN et al., 2016). Além disso, o uso da simulação realística proporciona ao aluno a vivência previa e possibilita o aprimoramento das habilidades práticas em um ambiente seguro, onde o estudante tem a oportunidade de errar sem trazer algum tipo de agravo para a saúde dos indivíduos (COSTA et al., 2017).

A simulação como método de ensino vem conquistando espaço em diversas universidades do mundo, tornando-se um recurso importante (ROBINSON BK e DEARMON V, 2013; BAPTISTA RCN, et al., 2014; KOPKA JÁ, et al., 2016; LIVINGSTON LL, et al., 2016).

A simulação pode ser compreendida como uma situação ou lugar criado de forma a possibilitar que um grupo de pessoas vivencie a representação e replicação de um acontecimento semelhante ao real, com o objetivo de praticar, aprender, avaliar ou compreender sistemas ou ações humanas (BRANDÃO CFS, COLLARES CF, MARIN HF, 2014).

Além disso, o uso da simulação como metodologia de ensino possibilita ao aluno uma melhor visualização da situação proposta, uma vez que, o aluno pode participar de forma ativa da situação replicada e assim, aprender com melhor exatidão o que foi proposto, já que, irá treinar suas habilidades técnicas, a gestão de crises, liderança, trabalho em equipe, discernimento clínico em circunstâncias críticas ou que possam provocar danos ao paciente (BRANDÃO CFS, COLLARES CF, MARIN HF, 2014).

Com isso, diante o despreparo prático dos alunos nos momentos de executar as técnicas, fez-se necessário a utilização da simulação realística, posto que, aproxima a realidade clínica através das situações criadas com os bonecos e cenários que replicam a realidade. O uso da simulação, por sua vez, possibilitará um grande avanço do conhecimento na área de saúde, tendo em vista que os estudos científicos apontam o uso da simulação como diferencial das outras metodologias de ensino devido à possibilidade da aprendizagem experiencial, focalizada no aluno em um ambiente seguro, respaldada por uma reflexão contínua e guiada por um professor (MARMOL et al., 2012; OLIVEIRA et al., 2014).

Nesse sentido o presente estudo tem como objetivo descrever a elaboração e construção de um vídeo para o ensino do exame físico respiratório para ser utilizado nas aulas de simulação clínica da disciplina de sistematização da assistência de enfermagem do curso de graduação em enfermagem. 


\section{RELATO DE EXPERIÊNCIA}

Trata-se de um estudo descritivo e metodológico em relação a elaboração e a construção de um vídeo de simulação para o ensino-aprendizagem do exame físico respiratório na disciplina de Sistematização da Assistência de Enfermagem, ofertada pelo curso de graduação em enfermagem de uma universidade pública localizada na região do triângulo mineiro.

O vídeo foi desenvolvido durante as atividades de extensão universitária de apoio ao laboratório de enfermagem a fim de serem implementadas junto aos alunos da disciplina mencionada anteriormente, no período de agosto de 2016 a janeiro de 2017. Participaram na elaboração do vídeo, duas alunas bolsistas do projeto de extensão, um aluno voluntário e ex-monitor da disciplina de Sistematização da Assistência de Enfermagem, um enfermeiro técnico do laboratório de enfermagem e dois docentes.

Esse processo de construção teve início com uma revisão bibliográfica sobre o uso da simulação no ensino da enfermagem e pela busca de um referencial teórico que fosse capaz de embasar as etapas subsequentes da construção dos vídeos.

Após estudo profundo sobre a construção e as técnicas envolvidas para a realização do exame físico respiratório, as alunas adaptaram um caso clínico através de um roteiro em que seria utilizado posteriormente durante as filmagens para a confecção das cens. O conteúdo foi gravado no período de uma semana e durante todo o processo as docentes estavam presentes para auxiliar as graduandas bolsistas na elaboração das técnicas. Após a gravação todo o material era analisado para detecção de erros ou falhas técnicas durante a execução das técnicas, feito isso, o vídeo era encaminhado para a edição e assim, utilizado pelos alunos da disciplina.

Foi sustentada pelas alunas a importância da elaboração de vídeos de simulação realística com as técnicas corretas do exame físico para o aprendizado e segurança dos alunos no campo prático, visto que, a insegurança destes pode acarretar em diversos erros que colocaram a segurança do paciente em risco. Além disso, o conhecimento aprofundado nas técnicas de inspeção, ausculta, percussão e palpação podem levar a detecção rápida de tórax retraído, qual o tipo de som emitido durante a percussão e até mesmo a presença de enfisema pulmonar durante a ausculta.

Aliado a isso, a utilização das tecnologias em saúde vinculadas com a metodologia ativa e o uso da simulação realística dentro das dependências da Universidade para o ensino aprendizagem trouxeram maior sustentação, segurança na aplicação das técnicas de enfermagem para a realização do exame físico respiratório no paciente. Uma vez que, os alunos sabem que possuem toda a estrutura e equipe capazes de fornecerem o melhor ambiente para o aprendizado.

Os princípios preconizados por Vygotsky LS (2007) levam em consideração a mediação, a comunicação, o diálogo, a interação, que são tidos como essenciais na estimulação de um pensamento crítico e de uma visão holística. Desta forma, foi eleita a abordagem histórica cultural como referencial teórico, pois vem de encontro com a metodologia ativa (VYGOTSKY LS, 2007).

Além do referencial de Vygotsky LS (2007) foi utilizado como modelo para elaborar o passo-a-passo do vídeo instrucional, o material didático de Barros ALBL (2015), conforme segue a seguir: O exame físico do sistema respiratório deve ser iniciado com a técnica da inspeção, de modo a avaliar a forma do tórax, as condições da pele, os movimentos respiratórios, a retração ou tiragem intercostal, a frequência respiratória, a amplitude da respiração, e o ritmo.

O passo seguinte é a técnica de palpação dos linfonodos cervicais, traqueia e tórax, cujo objetivo é avaliar a expansão e movimentos torácicos, frêmitos, massas e desvios. O próximo passo é a percussão, para determinar se o tecido pulmonar está cheio de ar ou líquido. Pode ser encontrado som claro pulmonar, hipersonoridade, som maciço, submaciço ou timpânico. O passo final é a ausculta, no qual é realizada a avaliação do fluxo de ar através da arvore brônquica, além da presença de líquidos ou obstruções no pulmão. Pode ser encontrado murmúrios vesiculares universalmente audíveis, murmúrios vesiculares diminuídos ou abolidos, sibilos, roncos, estertores, etc. 
Após, o aluno fez uma auto-avaliação, colocando em questão, os maiores desafios encontrados, os sentimentos vivenciados e como este conseguiu utilizar os conhecimentos adquiridos após essa metodologia ativa de ensino. O projeto de pesquisa foi encaminhado para a apreciação do Comitê de Ética em Pesquisa da Universidade Federal de Uberlândia e aprovado sob o número parecer ㄲo 1.864.940.

O vídeo de simulação para o ensino do exame físico respiratório foi construído, seguindo as etapas propostas. Foi cumprido o roteiro pré-estabelecido na metodologia e participação das alunas docentes na confecção do vídeo. Na elaboração e construção do vídeo foi possível verificar as seguintes potencialidades e fragilidades (Quadro 1).

Quadro 1 - Potencialidades e fragilidades encontradas durante a elaboração e construção de um vídeo de simulação para o ensino do exame físico respiratório.

\begin{tabular}{|l|l|}
\hline \multicolumn{1}{|c|}{ Potencialidades } & \multicolumn{1}{c|}{ Fragilidades } \\
\hline Motiva a criatividade & Dificuldade de recursos financeiros \\
\hline Estimula o raciocínio clínico & Falta de materiais e equipamentos \\
\hline $\begin{array}{l}\text { Proporciona um ambiente adequado para o diálogo e } \\
\text { as reflexões }\end{array}$ & Falta de tempo para desenvolver as filmagens \\
\hline $\begin{array}{l}\text { Permite um planejamento prévio e e } \\
\text { interdisciplinaridade. }\end{array}$ & $\begin{array}{l}\text { Falta de capacitação e preparo dos docentes e } \\
\text { discentes em relação as metodologias ativas }\end{array}$ \\
\hline Metodologias ativa de ensino & Modelo tradicional de ensino \\
\hline
\end{tabular}

Fonte: Cortes CF, Medeiros NAS, Silva PCS, Araújo, SA, Oliveira LF, Júnior, ERA, Deus KG, Dias DWO, 2019.

\section{DISCUSSÃO}

O objetivo do estudo foi descrever a experiência da construção de um vídeo de simulação para o ensino do exame físico, que sem dúvida é parte integrante do processo de assistência de enfermagem, sendo que para alcançar uma real aprendizagem em relação ao exame clínico é fundamental que os alunos sejam estimulados a construir seus próprios conhecimentos de forma que subsidiem sua vivência profissional.

O ensino de profissionais da saúde vem sendo, tradicionalmente, realizado por meio de aulas teóricas e práticas e pela consulta a materiais didáticos escritos como o livro. Entretanto, os questionamentos acerca da necessidade de novas metodologias para o ensino em saúde são frequentes e constituem preocupação contínua. (WILLIAMS P, et al. 2005). Por meio de um recurso audiovisual, o educando compreende de maneira sensitiva e não apenas cognitiva. Ao assistir um filme, além da transmissão de conteúdos, ocorrem vivencias de todos os tipos: emoções, sensações, atitudes, ações, conhecimentos etc. (ARROIO A, 2007).

Diversos estudiosos admitem a importância e relevância do uso de vídeos e outros recursos multimídia para tornar mais atraente e facilitar o processo de aprendizagem dos educandos (LIMA RP, 2010; CARNEIRO V, 1997; MIRANDA C E A e COPPOLA DGF, 2005; ANACLETO A, et al., 2007).A utilização de simulação e metodologias ativas no processo de formação dos alunos em enfermagem viabilizou a forma de pensar em relação ao processo de aprendizagem sob uma perspectiva de construção de saberes em que professores e estudantes se envolvem verdadeiramente (DAVID et al., 2019).

O uso da simulação no ensino do exame físico cria um espaço propício ao diálogo, a reflexão, a criatividade e a construção do saber (SANTOS MCD, et al., 2010; PARTECKE M, et al., 2016). No entanto, para a utilização desta estratégia é fundamental que os estudantes desenvolvam imagens reais dos pacientes (ROBINSON B K e DEARMON V, 2013; TERAOKA M, et al., 2016), sendo primordial a real compreensão do paciente e de seu estado de saúde.

A elaboração do vídeo de simulação busca sanar essa limitação por meio do treinamento em ambiente seguro e confiável, além de promover as habilidades psicomotoras relacionadas ao exame físico. A criação do vídeo permite que haja um espaço para o diálogo e o debate sobre as situações reais encontradas na prática cotidiana do profissional da saúde (PAI HC, 2016). 
Os estudos científicos apontam o uso da simulação clínica como diferencial das outras metodologias de ensino devido à possibilidade da aprendizagem experiencial, focalizada no aluno em ambiente seguro, respaldada por uma reflexão contínua, guiada por um professor (MARMOL MT, et al., 2012; OLIVEIRA SND, et al., 2014). Assim, o uso da simulação conduz o aluno a reflexão coletiva, ao diálogo e ao reconhecimento do contexto e de novas perspectivas, que são a base para a construção de novos caminhos, na busca pela integralidade.

Nossos achados corroboram com um trabalho realizado em 2015 com estudantes de graduação em Enfermagem de uma universidade federal do Nordeste, no qual diante da importância da aprendizagem significativa da realização do exame físico para se promover uma assistência de enfermagem sistematizada e eficiente, aplicou-se demonstração de vídeo e jogo educativo para os alunos do quarto período e obtiveram resultados positivos na dinâmica ensino-aprendizagem, confirmando a necessidade de aplicar técnicas de ensino mais dinâmicas, quebrando o padrão das metodologias tradicionais de ensino que de natureza são fracionadas e reducionistas (LIRA ALBC, et al., 2015)

Acredita-se, que a construção do vídeo, possibilitou a utilização de uma metodologia ativa e que busca por meio de uma prática reflexiva, crítica e comprometida promover a autonomia, a liberdade, o diálogo e a resiliência perante os conflitos.

\section{CONSIDERAÇÕES FINAIS}

A construção de um vídeo instrucional contribui de forma efetiva para o avanço da enfermagem e para a qualidade da assistência prestada, pois amplia as chances de contato dos discentes e profissionais de enfermagem com diferentes realidades a partir da utilização da simulação realística aliada ao uso das metodologias ativas para o ensino aprendizado. Além disso, auxiliam os alunos em relações as técnicas e habilidades no qual estas devem ser desenvolvidas, para que sejam feitas com segurança no ambiente de trabalho para que não se coloque a vida do paciente em risco.

\section{REFERÊNCIAS}

1. ALMEIDA RGDS, et al. Validation to Portuguese of the Scale of Student Satisfaction and Self-Confidence in Learning. Revista latino-americana de enfermagem, 2015; 23(6): 1007-1013.

2. ANACLETO A, et al. Cinema e Home Vídeo Entertaintnment: o mercado da magia e a magia do mercado, 2007.

3. ARROIO A. The role of cinema into science education. In: Science Education in a Changing Society. Lamanauskas, V. Siauliai: scientia Educologica, 2007.

4. BRANDÃO CF, COLLARES CF, MARIN HF. A simulação realística como ferramenta educacional para estudantes de medicina. Sci Med, 2014; 24(2): 187-92.

5. BAPTISTA RCN, et al. Students' satisfaction with simulated clinical experiences: validation of an assessment scale. Revista latino-americana de enfermagem, 2014; 22(5): 709-715.

6. BARROS ALBL. Anamnese e Exane Fisico: Avaliação Diagnostica de Enfermagem no Adulto. Porto Alegre: Artmed, 2015; 472p.

7. CARNEIRO V. O educativo como entretenimento na TV cultura. Um estudo de caso. Tese de doutorado, USP, 1997.

8. DAVID, F. S. et al. Evolution for emancipatory praxis: development of the realistic simulation method in undergraduate teaching in nursing. Online Brazilian Journal of Nursing, v. 17, n. 1, p. 13, 2019.

9. DE CARVALHO SANINO GE. O uso da simulação em enfermagem no Curso Técnico de Enfermagem. Journal of Health Informatics, 2012; 4.

10. JEFFRIES PR. A framework for designing, implementing, and evaluating: Simulations used as teaching strategies in nursing. Nursing education perspectives, 2005; 26(2): 96-103.

11. JOHNSEN HM, et al. Teaching clinical reasoning and decision-making skills to nursing students: Design, development, and usability evaluation of a serious game. International Journal of Medical Informatics, 2016; 94: 3948.

12. KOPKA JA, et al. Helping Students Process a Simulated Death Experience: Integration of an NLN ACE. S Evolving Case Study and the ELNEC Curriculum. Nursing Education Perspectives, 2016; 37(3): 180-182.

13. LIMA R P. O vídeo na sala de aula: breve reflexão a partir das contribuições de Mário Kaplún e Paulo Freire, 2010.

14. LIVINGSTON LL, et al. Simulated Disaster Day: Benefit From Lessons Learned Through Years of Transformation From Silos to Interprofessional Education. Simulation in Healthcare, 2016; 11(4): 293-298.

15. LIRA ALBC, et al. Estratégia de Aprimoramento do Ensino do Exame Físico. Enfermagem em Foco, $2015 ; 6$,: 57-61. 
16. MARMOL MT, et al. Central catheter dressing in a simulator: the effects of tutor's assistance or self-learning tutorial. Revista latino-americana de enfermagem, 2012; 20(6): 1134-1141.

17. MIRANDA C E A; COPPOLA D G F. A educação pelo cinema. Rev. Educação e Cinema, 2005: 02.

18. OLIVEIRA SND, et. Utilização da simulação no ensino da enfermagem: revisão integrativa. Revista Mineira de Enfermagem, 2014; 18(2): 487-504.

19. PAI HC. Development and validation of the Simulation Learning Effectiveness Scale for nursing students. Journal of Clinical Nursing, 2016; 25(21-22): 3373-3381.

20. PARTECKE M, et al. Interprofessional Learning-Development and Implementation of Joint Medical Emergency Team Trainings for Medical and Nursing Students at Universitätsmedizin Greifswald. GMS Journal for Medical Education, 2016; 33(2).

21. ROBINSON BK, DEARMON V. Evidence-based nursing education: Effective use of instructional design and simulated learning environments to enhance knowledge transfer in undergraduate nursing students. Journal of Professional Nursing, 2013; 29(4): 203-209.

22. SANTOS MCD, et al. Recontextualization of Nursing clinical simulation based on Basil Bernstein: semiology of pedagogical practice. Revista Gaúcha de Enfermagem, 2010; 31(4): 746-752.

23. TERAOKA $M$, et al. Design of a Program to Learn the Nursing Process by Introducing Digital Stories-For the Promotion of Patient Understanding. Studies in health technology and informatics, 2016; 225: 965.

24. VYGOTSKY LS. A formação social da mente. São Paulo: Martins Fontes, 2007; 224p.

25. WILLIAMS P, et al. E-learning: what the literature tell us about distance education. An overview. Aslib proccedings: new information perspectives, 2005; 57: 109-22. 